\title{
Pontos de abastecimento de pulverizadores agrícolas: U ma revisão comparando os modelos em uso
}

\begin{abstract}
Luciano G ebler ${ }^{1}$
RESU M O

Pontos de abastecimento de pulverizadores são locais destinados ao preparo das caldas agroquímicas e carregamento de equipamentos de pulverização agrícola, classificados pela legislação ambiental e trabalhista como equipamento de segurança ambiental. Há suficiente bibliografia técnica que recomenda e descreve os processos construtivos e de manejo de tais locais, mas há pouca clareza sobre como se chegou a esta forma consensual, aliado ao pouco embasamento científico de seus benefícios e riscos agregados. Alguns aspectos como impermeabilização, constituição do material, manejo do piso e dos resíduos de agroquímicos, interferem na capacidade de evitar que os possíveis contaminantes atinjam 0 solo e as águas, interferindo no descarte final. Aspectos funcionais ainda carecem de respostas, como a possibilidade do piso reter e armazenar o contaminante e se tornar um emissor a longo prazo, a definição do tempo de vida útil do equipamento, dentre outros. Recentemente houve avanços na filosofia construtiva dos pontos de abastecimento de pulverizadores, com a introdução da remediação de resíduos in situ, através da biodegradação, ganhando denominações, como biobeds, dentre outras. 0 objetivo desta revisão é discutir as diferentes formas de pisos e manejo nestes locais, seus riscos e a possibilidade de se tornarem um passivo ambiental.
\end{abstract}

Palavras-chave: Biobed, pisos para manuseio de agrotóxicos, contenção de contaminantes

\section{Agricultural chemical handling pads: A review comparing the models in use}

\begin{abstract}
Sprayers handling pads, among other designations, are described as sites intended for the preparation of pesticides and load of agricultural pulverization equipments. By the environmental and labour legislation, these points are seen as environmental security equipment in the rural surroundings (environment). There is enough technical bibliography that describes the constructive and handling processes of such sites, but there is insufficient clarity about how this consensual manner was reached, with little scientific basis about its collective advantages and risks. Some aspects such as the impermeabilization, material formation, management of floor and residue of agrochemicals, interfere in the capacity of preventing possible polluters reaching the soil, surface and groundwater, also influencing its final disposal. Some functional aspects still require answers, such as possibility of the floor retaining and storing the pollutant and becoming a emitter in long-term, the definition lifetime equipment, amongst others. Recently there have been advances in the constructive philosophy of sprayers handling pads, with the introduction of residue redressing in situ, through biodegradation which became know $\mathrm{n}$ as biobeds, amongst others. The objective of this revision is to discuss the different types of floor bads and management of these places, its risks and possibilities of becoming environmental liabilities.
\end{abstract}

Key words: Biobed, floors for handling of agrochemicals; contaminant containment 


\section{INTRODUÇÃO}

Os pontos de abastecimento de agrotóxicos (PAAs), no meio rural, têm sido tratados como parte das Boas Práticas Agrícolas (BPAs), e, na maioria das vezes, suas informações têm sido apresentadas na forma de manuais técnicos locais ou páginas de Internet (FAO, 2001; DEFRA, 2006). Mesmo assim, há poucos estudos científicos publicados sobre como se chegou às atuais características estruturais.

Apesar da evolução das ciências agrícolas, houve pouca alteração na estrutura básica dos locais onde é feito o abastecimento de pulverizadores de agroquímicos, composta de um ponto de fornecimento de água e um espaço impermeável grande o suficiente para o manejo do equipamento (Huber et al., 2000). Tradicionalmente, a variação entre as recomendações para estes locais se resumia na composição do material do piso e a maneira de construí-lo.

Nestes PAAs, ocorre um processo antagônico ao planejado para o uso de agrotóxicos em áreas agrícolas (Senseman et al., 1996). Tais produtos são testados e autorizados para uso na forma de pequenas doses distribuídas em uma grande área de terreno ou em um grande volume de água, resultando em concentrações de $\mathrm{g} \mathrm{ha}^{-1}$ ou $\mathrm{mg} \mathrm{L}^{-1}$ de ingrediente ativo (Castillo et al., 2008). Sob o aspecto ambiental, a esta forma de trabalho é conferido o termo contaminação difusa, ou não-pontual.

Quando se trabalha com agrotóxicos concentrados (comercial), sempre no mesmo ponto e repetidamente num curto período de tempo, ocorre a contaminação pontual, produzindo concentrações equivalentes a gramas ou decigramas por metro quadrado (Ramwell et al., 2004).

Isto aumenta o risco de que o agrotóxico possa ultrapassar o limite de concentração recomendada para a segurança ambiental, o que deve ser evitado constantemente (Andréa et al., 2004; Reichemberger et al., 2007).

O objetivo desta revisão é discutir as diferentes formas de pisos e estruturas destes pontos de abastecimento, seus riscos e possibilidade de se tornarem um passivo ambiental na propriedade rural, comparando entre os dois modelos atualmente em uso no mundo.

\section{SOLUÇÕES ADOTADAS QUANTO À FILOSOFIA DO MANEJO DO PISO E DO RESÍDUO}

Sob o aspecto ambiental, a principal função do local de abastecimento é impedir os contaminantes de atingirem pontos passíveis de danos ambientais ou causarem risco a uma população (Wenneker et al., 2008).

Desconsiderando as tentativas de utilização de impedimento natural (leito de rocha ou argila compactada), que por sua natureza, não garantem segurança ao ponto de abastecimento por falta de controle construtivo, há hoje duas formas de se manejar o resíduo de agrotóxico nestes locais.

O primeiro modelo, ou sistema, é baseado na idéia de coleta e descarte. Assim, constrói-se um local que possibilite o recolhimento de eventuais derrames de agrotóxicos e seus resíduos, dispondo-os posteriormente em local apropriado. Isto é feito através da impermeabilização e da conformação do piso, usando materiais como concreto (Noyes, 1989; Gebler, 2004), ou aço (Noyes et al., 1996), evitando que o contaminante chegue ao solo e às águas.

Neste sistema, dependendo do material escolhido para o ponto de abastecimento, existe o risco físico relacionado com a quebra da impermeabilização, incrementando a velocidade ou o volume no transporte de contaminantes em direção ao solo e subsolo (Carter, 2000).

Isto pode acontecer, por exemplo, pela formação de rotas preferenciais (rachaduras) através da pressão exercida pelos rodados das máquinas agrícolas, ou por abrasão química, provocada pela natureza físico-química dos produtos reagindo com o material construtivo utilizado (Noyes, 1989; Helweg et al., 2002). Em ambos os casos, são variáveis dependentes, que demandam estudos relacionados com a ciência dos materiais.

Já a retirada do excesso de contaminantes da superfície, utilizando-se ou não o princípio da tríplice lavagem do piso, objetiva a redução da taxa de risco (Ramwell et al., 2004; Reichenberger et al., 2007).

Isto é necessário, pois a configuração do ponto de descarte apropriado para este resíduo também se encontra em discussão em muitos locais, não havendo, para tanto, recomendação científica uniforme e nem clareza na legislação brasileira (ABNT, 2004).

A questão do descarte no Brasil pode ser resumida a dois problemas: i) Concentração de resíduos em relação à concentração de trabalho e, ii) o impedimento legal da mistura destas sobras de agrotóxicos com outras substâncias sem o manejo apropriado. Esta última preocupação encontra respaldo na legislação brasileira, ao proibir misturas de produtos comerciais diferentes nos tanques de pulverização sem prévia aprovação governamental.

A legislação determina, ainda, o momento em que a sobra do agrotóxico passa a ser classificada como resíduo perigoso, saindo do âmbito da Lei dos agrotóxicos, para as interpretações da Lei de crimes ambientais e resoluções do Conselho Nacional de Meio Ambiente, que regem este assunto segundo a natureza do composto resultante.

Portanto, não havendo mistura, e seguindo basicamente a interpretação técnica, a solução para o resíduo fica contida no âmbito da Lei dos agrotóxicos, devendo-se prever sua destinação como tal (Gebler, 2004).

Assim, sobre a superfície dos PAAs, mesmo o contaminante sofrendo o mesmo tipo de ação de degradação que ele encontra no solo, a proporção desta pode ser diferenciada, muitas vezes, com intensidades ou velocidades de reação exponencialmente inferior a do processo natural de atenuação, pois, sobre esta superfície artificial, há grande probabilidade que predominem reações não biológicas (Fay et al., 1997).

Esta menor taxa esperada de biodegradação, em relação às demais rotas existentes, é devido à natureza observada do local, já que vida microbiana expressiva, responsável pela maior eficiência na remediação de áreas impactadas com moléculas orgânicas, tem dificuldade em se manter na superfície exposta do ponto de abastecimento (Corseuil \& Alvarez, 1996; Monteiro, 1997). 
Ainda hoje há algumas lacunas de conhecimento sobre a metodologia no trato de resíduos.

Discute-se, por exemplo, sem a necessária precisão, sobre o que acontece quando há a formação de rachaduras no piso de concreto que permitam ao contaminante se afastar da superfície e da luz solar (diminuindo ou eliminando os processos de fotólise e volatilização), somado ao retardo da ação biodegradadora devido à possível baixa população biótica nestes locais (Carter, 2000; Helweg et al., 2002).

Nestes casos, dependendo da natureza química do agrotóxico e dos compostos do concreto, é possível que o piso do ponto de abastecimento de pulverizadores passe a se comportar como um reservatório de acumulação (Ramwell, 2005), garantindo a integridade da molécula de contaminante naquele ponto.

Com base no incremento recente do conhecimento de biodegradação dos diversos agrotóxicos, surgiu o segundo modelo ou sistema de trabalho no desenho dos PAAs. Passouse a sugerir a utilização do local como um reator biológico in situ, denominado inicialmente de biobed (Torstensson, 2000; Castillo et al., 2008; Rose et al., 2008).

O primeiro objetivo ambiental do reator (biobed) é o mesmo do piso impermeável, evitar que os contaminantes atinjam solo e água, reduzindo riscos de contaminação ambiental.

Além disso, ao sugerir que o tratamento destes resíduos seja feito no próprio local, através de um processo biológico de leito fíxo, ele tenta responder a questão de inativação local do resíduo, onde todo o volume de líquidos que é derrubado durante o processo de enchimento do pulverizador é retido em um composto organo-mineral, que privilegia ações de biorremediação.

Este composto apresenta uma vida útil determinada pelo seu uso, sendo recomendada sua substituição no momento que sua massa é reduzida (determinado pela altura da coluna de substrato no biobed) (Spliid et al., 2006; Fait et al., 2007; Vischetti et al., 2008; Monaci et al., 2009). Em países da Europa, que recomendam ou adotam o biobed como mecanismo de segurança nos pontos de trabalho com pulverizadores, os testes demonstraram que, uma vez cumpridas as exigências construtivas e de manejo do sistema, há a inativação de todos os resíduos, mesmo os mais resilientes (Torstensson \& Castillo, 1997; Fogg et al., 2003a; 2003b; 2004a; Gregoire et al., 2008). Esta inativação se dá tal qual a biodegradação existente no solo, porém é artificialmente acelerada pelo fornecimento de matéria orgânica fresca e aeração em profundidade, que favorece aos processos de degradação biológica (Vischetti et al., 2004). Portanto, a mistura para preenchimento do biobed (solo, dejetos orgânicos animais e de palha), também conhecida como mix biológico, favorece a redução do movimento vertica (percolação)1 do contaminante, pela adsorção das moléculas de agrotóxico, e a disponibilização facilitada dos contaminantes como substrato às bactérias e fungos do sistema (Castillo \& Torstensson, 2007). Assim é feito a contenção e o tratamento final dos resíduos in situ, onde, após o tempo de vida util do mix, deve-se ainda compostar a massa por um período de segurança fora do biobed, utilizando-a posteriormente como adubação orgâno-mineral suplementar na propriedade rural (Debaer \& Jaeken, 2006; Copolla et al., 2007).
As limitações apontadas para este modelo são: A) a necessidade de controle no fluxo de água de entrada, que deve permanecer abaixo de determinada velocidade de percolação, evitando acúmulo nas camadas inferiores do biobed, que dificultaria o fluxo de ar por todo o sistema (Fogg et al., 2004b); B) a questão referente à segurança do ambiente, uma vez que o biobed é desenhado para tratar de gotejamentos, vazamentos ou leves derrames acidentais que ocorram no momento do trabalho de enchimento do pulverizador, mesmo tendo havido poucos testes que reproduzissem situações limites, como em casos de derrame de grandes volumes de produto comercial concentrado de agrotóxico (Helweg et al., 2002); C) o custo de manutenção do biobed pode ser outro fator limitante, uma vez que ao envolver mão de obra da propriedade rural, para manejo do biomix, gerando custos extras em relação aos outros modelos (Castillo et al., 2008).

\section{AsPeCTOS CONSTRUTIVOS DOS DIFERENTES MODELOS DE PISOS DE PONTOS DE ABASTECIMENTO}

Quanto aos aspectos construtivos, ambos os modelos apresentam uma exigência em comum: Impermeabilização (ou isolamento). Mesmo para os biobeds, a impermeabilização da caixa que contém o mix biológico é uma medida de segurança recomendada (Castillo et al., 2008; Rose et al., 2008).

No modelo baseado em pisos para os pontos de abastecimento, agregado à segurança dada pela impermeabilização, deve-se acompanhar ainda de um reforço estrutural adequado ao esforço exercido nos pontos de contato dos rodados dos tratores e implementos sobre toda a superfície (Gebler, 2004; Rufatto et al., 2006). Este reforço, necessário para fazer frente às forças de tração e compressão, será dependente do material que compõe o piso.

Construtivamente a decisão de espessura, qualidade, uso uniforme ou misto de materiais, reforço estrutural, irá depender de fatores como trânsito, peso dos tratores e equipamento, tipo de agroquímicos utilizados, período úmido no ano, dentre outros.

O material que melhor responde a estes critérios é o metal, e dentre eles, o aço (Noyes et al., 1996). É um material uniforme, cujas seções devem ser impermeabilizadas por soldas estanques, aplicado na forma de piso integral ou como camada de revestimento sobre outro material (de origem reciclada ou não), sem perda de eficiência. Além disso, suporta as trações e pressões exercidas pelas forças externas.

Na maioria das vezes, dependendo do mercado local, o fator negativo relacionado ao uso do aço é o custo elevado, dependente da qualidade exigida para fazer frente à corrosão química e hídrica, que ditará o tempo de vida do revestimento do piso.

Estruturalmente a decisão de espessura, uso uniforme ou misto, reforço estrutural e qualidade do material irá depender de fatores como trânsito, peso dos tratores e equipamento, tipo de agroquímicos utilizados, período úmido no ano, dentre outros. 
O concreto tem sido utilizado como o principal material construtivo dos pontos de abastecimento de pulverizadores, isoladamente ou em conjunto com os revestimentos de aço (Noyes et al., 1996).

Quando novo, apresenta suficiente impermeabilização e resistência moderada aos esforços, necessitando reforço de armadura estrutural, porém mantendo um custo acessível (Gebler, 2004).

$\mathrm{O}$ atendimento aos critérios de segurança por parte deste material irá depender de fatores construtivos como: a) tipo de cimento (FCK) e dos agregados (areia e brita), e seu manejo, pois todos influem no volume de poros internos; b) espessura do piso; c) diâmetro da ferragem e dimensão da trama da armadura; d) forma de impermeabilização utilizada (física ou química), dentre outros (Helene, 1997; ABNT, 2003).

Alem disso, dependerá de fatores antrópicos externos, como volume de uso do local, espécie química do material usado, peso dos equipamentos (vinculado com o tipo de rodado), dentre outros.

Os aspectos negativos no uso do concreto armado como material do piso é a eventual formação de rotas preferenciais (rachaduras) e excessiva degradação química, principalmente em situações de condições adversas, facilitando a percolação de líquidos.

Ainda, dependendo de fatores construtivos (materiais, técnicas e mão-de-obra inadequada), o concreto pode se apresentar demasiadamente poroso, absorvendo os contaminantes da parte superior e os liberando, após determinado período, em sua parte inferior. Havendo esta ação, ele passa da classificação de equipamento de segurança para fonte emissora para passivo ambiental.

Assim, a vida útil do piso de concreto armado irá depender de vários aspectos, como composição, construção e manejo, sendo necessários estudos sobre a interação entre os agentes químicos dos agrotóxicos com os componentes do concreto (além do cimento e agregados, pode-se esperar a presença de aditivos).

Um dos fatores recomendados que possa influenciar positivamente estas rampas no meio rural, seria a procura por profissionais habilitados para o cálculo estrutural, dimensionando os elementos da construção segundo projeto (Noyes, 1989)

Alguns outros materiais, como lonas plásticas ou polímeros plásticos aplicados sobre o solo, foram cogitados como impermeabilizantes de piso, mas ainda não demonstraram reunir as condições necessárias de resistência, impermeabilidade ou durabilidade para prover este local com a segurança exigida. (Cooper \& Taylor, 2008)

Em relação aos biobeds, as necessidades estruturais quanto a resistência de esforços se concentram no suporte aos trilhos de movimentação do maquinário agrícola e na resistência das paredes da caixa de retenção às forças laterais provindas do solo (Rose et al., 2008).

Quanto a impermeabilização do fundo do biobed, ou mesmo de suas paredes, por não haver transito direto de máquinas, além de aço ou concreto, pode-se lançar mão de materiais alternativos, como lonas plásticas ou geomembrana (Castillo et al., 2008). Isto pode resultar em um custo construtivo menor, porém com uma vida útil proporcionalmente mais curta que em relação aos materiais tradicionais.

Em relação ao custo, um dos fatores que também oneram o biobed é a necessidade de mão de obra treinada para sua construção, preparo e colocação inicial do mix de compostagem, necessitando-se treinar e manter uma equipe antes da implantação do sistema.

\section{UMA SOLUÇ̃̃O INTERMEDIÁRIA?}

Quando se avalia cada sistema ou material isoladamente, surgem pontos fortes e fracos para cada um. Recentemente tem sido sugerida a reunião de materiais ou processos de forma complementar.

O que se busca é um cenário ideal, com a garantia da segurança quanto ao controle de fluxo e coleta em derrames volumosos dado pelos pisos de aço e a ação local de biorremediação encontrada nos biobeds.

Nesta linha, alguns sistemas mistos de biorreatores acoplados a pisos de abastecimento de pulverizadores, como o biomassbed, Phytobac e o biofiltro, já vem sendo testados (Vischetti et al., 2006; Wilde et al., 2007).

Estes modelos apresentam alterações de estrutura em relação aos modelos básicos discutidos anteriormente, mas, como apontado por estes estudos, o problema para adoção destes métodos está relacionado principalmente aos custos de construção e ao acréscimo de mão de obra para o manejo dos biorreatores, dependendo sua implantação de incentivos externos ou obrigações governamentais.

\section{CONSIDERAÇÕES FINAIS}

1. Pontos de abastecimento de pulverizadores bem estruturados são equipamentos de segurança ambiental e do trabalhador extremamente necessários, com a função de contenção de derrames acidentais e posterior tratamento dos eventuais resíduos.

2. No Brasil há pouca preocupação sobre os reais efeitos da contaminação pontual provocada por agrotóxicos e seus resíduos em pontos de abastecimento de pulverizadores, sendo a maioria das construções baseadas em recomendações empíricas.

3. Além da pouca informação relacionada aos aspectos construtivos, faltam estudos referentes à interação entre os diversos compostos químicos com os materiais que tradicionalmente compõe os pontos de abastecimento de pulverizadores, o que pode influenciar a vida útil e o custo da estrutura.

4. Faltam estudos da eficiência dos bioreatores/biobeds para manejo e descarte final de resíduos de agrotóxicos em condições brasileiras.

5. Há pouca clareza das leis brasileiras sobre o assunto, com excesso de regulação em alguns pontos e absoluta falta 
de menção de aspectos técnicos vinculados aos pontos de abastecimento e descarte de resíduos em outros.

\section{Agradecimentos}

Agradecimentos ao Prof. Ronald Noyes, por sua pronta colaboração no fornecimento de documentos particulares para esta revisão bibliográfica e à Dra. Maria del Pillar Castillo, pela sua colaboração na busca pela resolução deste problema.

\section{LITERATURA CITADA}

Andréa, M. M.; Papini, S.; Peres, T. B.; Bazarin, S.; Savoy, V. L. T.; Matallo, M. B. Glyphosate: influência na bioatividade do solo e ação de minhocas sobre sua dissipação em terra agrícola Planta Daninha, v.22, p.95-100. 2004.

ABNT - Associação Brasileira de Normas Técnicas. NBR 6118: Projeto e Execução de Obras de Concreto Armado. Rio de Janeiro, 2003. 225p.

ABNT - Associação Brasileira de Normas Técnicas. NBR 10004: Classificação de Resíduos. Rio de Janeiro, 2004. 71p.

Carter, A. How pesticides get into water-and proposed reduction measures. Pesticide Outlook, v.11, p.149-156, 2000.

Castillo, M. del P.; Torstensson, L. Effect of biobed composition, moisture, and temperature on the degradation of pesticides. Journal of Agricultural Food Chemistry, v.55, p.5725-5733, 2007.

Castillo, M. D. P.; Torstensson, L.; Stenström, J. Biobeds for environmental protection from pesticide use - A review. Journal of Agriculture and Food Chemistry, v.56, p.62066219, 2008.

Cooper, S. E.; Taylor, W. A. Techniques and hardware to reduce point source pollution of water with pesticides; a UK TOPPS perspective on predicted current practice and where future training may need emphasis-project. Aspects of Applied Biology, v.84, p.385-394, 2008.

Coppola, L.; Castillo, M. del P.; Monaci, E.; Vischetti, C. Adaptation of the biobed composition for chlorpyrifos degradation to Southern Europe conditions. Journal of Agricultural Food Chemistry, v.55, p.396-401, 2007.

Corseuil, H. X.; Alvarez, P. J. J. Natural bioremediation perspective for BTX-contaminated groundwater in Brazil. Water Science and Technology, v.34, p.311-318, 1996.

Debaer, C.; Jaeken, P. Modified biofilters to clean up leftovers from spray loading and cleaning; experience from pilot installations. Aspects of Applied Biology, v.77, p.247-252, 2006.

DEFRA - Department for Environment, Food and Rural Affairs. Code for pratice for using plant protection products.http:// www.pesticides.gov.uk/farmers_growers_home.asp. 16 Jun. 2008.

Fait, G.; Nicelli, M.; Fragoulis, G.; Trevisan, M.; Capri, E. Reduction of point contamination sources of pesticide from a vineyard farm. Environmental Science \& Technology, v.41, p.3302-3308, 2007.
FAO - Food and Agriculture Organization. Guidelines on good practice for ground application of pesticides. http:// www.fao.org/DOCREP/006/Y2767E/Y2767E00.HTM\#3. 16 Jun. 2008 .

Fay, E. F.; Silva; C. M. M. S.; Melo, I. S. Degradação abiótica de xenobióticos. In: Melo, I. S. (org.). Microbiologia ambiental. Jaguariúna: EMBRAPA, 1997. p.125-140.

Fogg, p.; Boxall A. B.A.; Walker, A.; Jukes A. Pesticide degradation in a 'biobed' composting substrate. Pest Management Science, v.59, p.527-537, 2003a.

Fogg, P.; Boxall, A. B.A.; Walker, A.; Jukes, A. Degradation of pesticides in biobeds: The effect of concentration and pesticide mixtures. Journal of Agricultural Food Chemistry, v.51, p.5344-5349, 2003 b.

Fogg, P.; Boxall A. B. A.; Walker A.; Jukes A. Degradation and leaching potential of pesticides in biobed systems. Pest Management Science, v.60, p.645-654, 2004a.

Fogg, P.; Boxall, A. B. A.; Walker, A.; Jukes, A. Leaching pesticides from biobeds: Effect of biobed depth and water loading. Journal of Agricultural and Food Chemistry, v.52, p.6217-6227, 2004b.

Gebler, L. Recomendações para a construção, manutenção e segurança de pontos de abastecimento de pulverizadores para a produção integrada de maçãs no Brasil. 2 ed. Bento Gonçalves: Embrapa Uva e Vinho, 2004. 13p.

Gregoire, C.; Elsaesser, D.; Huguenot, D.; Lange, J.; Lebeau, T.; Merly, A.; Mose, R.; Passeport, E.; Payraudeau, S.; Shütz, T.; Schulz, R.; Tapia-Padilla, G.; Tournebize, J.; Trevisan, M.; Wanko, A. Mitigation of agricultural nonpoint-source pesticide pollution in artificial wetland ecosystems. Environmental Chemistry Letters, v.7, p.205-231, 2008.

Helene, P.R.L. Introdução da durabilidade no projeto das estruturas de concreto. In: workshop durabilidade das construções, 1997, São Leopoldo. Anais... São Leopoldo: ANTAC, 1997. p.31-42.

Helweg, A.; Bay, H.; Hansen, H. P. B.; Rabølle, M.; Sonnenborg, A.; Stenvang, L. Pollution at and below sites used for mixing and loading of pesticides. International Journal of Environmental Analytical Chemistry, v.82, p.583-590, 2002.

Huber, A.; Bach, M.; Frede, H. G. Pollution of surface waters with pesticides in Germany: Modeling non-point source inputs. Agriculture, Ecossystems and Environment, v.80, p.191-204, 2000.

Monaci, E.; Coppola, L.; Casucci, C.; Perucci, P.; Vischetti, C. Retention capacity of an organic bio-mixture against different mixtures of fungicides used in vineyards. Journal of Environmental Science and Health Part B, v.44, p.724-729, 2009.

Monteiro, R. T. R. Degradação de pesticidas. In: Melo, I. S. (org.). Microbiologia ambiental. Jaguariúna: EMBRAPA, 1997. p.107-124.

Noyes, R. I. Modular farm sized concrete agricultural chemical handling pads. [S.1.]: Oklahoma City: Oklahoma State University, Agricultural Engineering Department, 1989. 22p.

Noyes, R.T.; Gardisser, D. R.; Kammel, D. W. Steel containment and mix/load pad construction guidelines. In: Pesticide and fertilizer containment seminar, 1996, Oklahoma City. Fact sheets, 1996. 10p. 
Ramwell, C.T. Herbicide sorption to concrete and asphalt. Pest Management Science, v.61, p.144-150, 2005.

Ramwell, C.; Johnson, P. D.; Boxall, A. B. A.; Rimmer, D. Pesticide residues on the external surfaces of field-crop sprayers: Environmental impact. Pest Management Science, v.60, p.795-802, 2004.

Reichenberger, S.; Bach, M.; Skitschak, A.; Frede, H. G. Mitigation strategies to reduce inputs into ground - and surface water and their effectiveness; A review. Science of the Total Environment, v.384, p.1-35, 2007.

Rose, S.; Basford, B.; Carter, A. Development of a design manual for agricultural pesticide handling and washdown areas. www.environment-agency.gov.uk. 16 jun. 2008.

Senseman, S. A.; Lavy, T. L.; Daniel, T. C. Monitoring groundwater for pesticides at selected mixing/loading sites in Arkansas. Environmental Science and Technology, v.31, p 283-288. 1996.

Spliid, N. H.; Helweg, A.; Heinrichson, K. Leaching and degradation of 21 pesticides in a full-scale model biobed. Chemosphere, v.65, p.2223-2232, 2006.
Torstensson, L. Experiences of biobeds in practical use in Sweeden. Pesticide Outlook. v.11, p.206-211, 2000.

Torstensson, L.; Castillo, M. del P. Use of biobeds in Sweden to minimise environmental spillages from agricultural spray equipment. Pesticide Outlook, v.8, p.24-27, 1997.

Vischetti, C.; Capri, E.; Trevisan, M.; Casucci, C.; Perucci, P. Biomassbed: A biological system to reduce pesticide point contamination at farm level. Chemosphere, v.55, p.823-828, 2004.

Vischetti, C.; Monaci, E.; Cardinali, A.; Perucci, P. The effect of initial concentration, co-application and repeated applications on pesticide degradation in a biobed mixture. Chemosphere, v.72, p.1739-1743, 2008.

Wenneker, M.; Beltman, W. H. J.; Werd, H. A. E.; van de Zande, J. C. Identification and quantification of point sources of surface water contamination in fruit culture in the Netherlands. Aspects of Applied Biology, p.369-376, 2008.

Wilde, T. de; Spanoghe, P.; Debaer, C.; Ryckeboer, J.; Springael, D.; Jaeken, P. Overview of on-farm bioremediation systems to reduce the occurrence of point source contamination. Pest Management Science, v.63, p.111-128, 2007. 